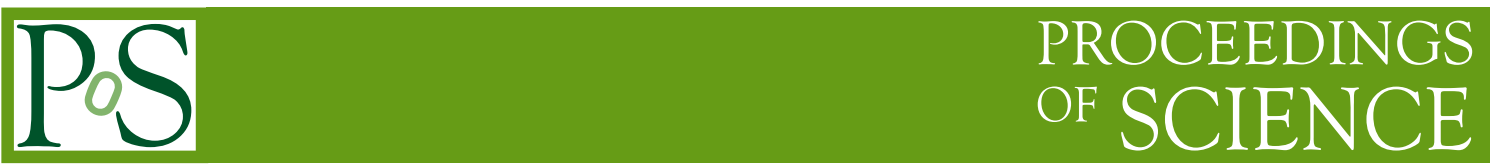

\title{
High resolution X-ray Timing from a LOFT
}

\author{
I. Donnarumma ${ }^{* a}$, R. Campana ${ }^{a}$, L. Stella ${ }^{b}$, G. L. Israel ${ }^{b}$, M. $\operatorname{Feroci}^{a}$, T. Belloni ${ }^{c}$, \\ S. Campana ${ }^{c}$, E. Costa $^{a}$, E. Del Monte ${ }^{a}$, Y. Evangelista ${ }^{a}$, C. Labanti ${ }^{d}$, F. Muleri $^{a}$, \\ M. Rapisarda ${ }^{a, e}$, A. Rashevsky ${ }^{f}$, A. $\operatorname{Vacchi}^{f}$, G. Zampa ${ }^{f}$, N. $\operatorname{Zampa}^{f}$, P. Attinà ${ }^{g}$, \\ G. Baldazzi ${ }^{h}$, G. Bertuccio ${ }^{i}$, V. Bonvicini ${ }^{f}$, E. Bozzo ${ }^{j}$, L. Burderi ${ }^{k}$, A. Corongiu ${ }^{l}$, \\ S. Covino ${ }^{c}$, S. Dall'Osso ${ }^{b}$, D. De Martino ${ }^{m}$, T. Di Salvo ${ }^{n}$, F. Fuschino $^{d}$, M. Grassi $^{o}$, \\ F. Lazzarotto ${ }^{a}$, P. Malcovati ${ }^{o}$, M. Marisaldi ${ }^{d}$, S. Mereghetti ${ }^{p}$, M. Orio $^{q, r}$, A. Pellizzoni ${ }^{l}$, \\ L. Pacciani ${ }^{a}$, A. Papitto ${ }^{k}, l$, L. Picolli ${ }^{o}$, P. Porta ${ }^{g}$, A. Possenti ${ }^{l}$, P. Soffitta ${ }^{a}$, R. Turolla ${ }^{s}$, \\ L. Zampieri ${ }^{r}$ \\ a INAF/IASF-Roma \\ ${ }^{b}$ INAF/Osservatorio Astronomico di Roma \\ ${ }^{c}$ INAF/Osservatorio Astronomico di Brera \\ ${ }^{d}$ INAF/IASF-Bologna \\ e ENEA-Frascati \\ ${ }^{f}$ INFN/Sezione di Trieste \\ $g$ Thales Alenia Space Italia Torino \\ ${ }^{h}$ Università di Bologna, Dip. di Fisica \\ ${ }^{i}$ Politecnico di Milano Dip. di Ingegneria Elettrica \\ ${ }^{j}$ ISDC \\ Chemin dÕEcogia 16, CH-1290, Versoix, Switzerland \\ ${ }^{k}$ Università di Cagliari, Dip. di Fisica \\ ${ }^{l}$ INAF/Osservatorio Astronomico di Cagliari \\ ${ }^{m}$ INAF/Osservatorio Astronomico di Capodimonte \\ ${ }^{n}$ Università di Palermo, Dip. di Fisica \\ o Università di Pavia, Dip. di Ingegneria Elettrica \\ p INAF/IASF-Milano \\ ${ }^{q}$ INAF/Osservatorio Astronomico di Torino \\ ${ }^{r}$ INAF/Osservatorio Astronomico di Padova \\ ${ }^{s}$ Università di Padova, Dip. di Fisica \\ E-mail: immacolata.donnarummadiasf-roma.inaf.it
}


LOFT (Large area Observatory For X-ray Timing) is an innovative mission concept for a next generation of X-ray timing experiments. Recent developments in the field of Silicon detectors allow to design a realistic observatory devoted to X-ray timing studies with an effective area around $13 \mathrm{~m}^{2}$, in the energy range $2-30 \mathrm{keV}$. In this paper we present the mission concept together with the key properties of the detectors, as demonstrated in our laboratory, including an energy resolution better than $500 \mathrm{eV}$ at room temperature. The overall power and weight budgets fit to a standard mission design. Such an exceedingly large area ( $>20$ times RXTE/PCA), with a time resolution better than $10 \mu \mathrm{s}$, will allow unprecedently fast and accurate time variability studies related to accreting compact objects (e.g. fast coherent pulsations and QPOs). We describe here the scientific performance of a timing observatory in the $10 \mathrm{~m}^{2}$ class for open problems in fundamental physics, such as strong gravity effects, the measurement of the mass of black holes and neutron stars, the equation of state of ultradense matter. Finally, we present the results of simulated LOFT observations.

High Time Resolution Astrophysics (HTRA) IV - The Era of Extremely Large Telescopes May 5 - 7, 2010

Agios Nikolaos, Crete Greece

\footnotetext{
* Speaker.
} 


\section{Introduction}

In the last years important progress in the field of General Relativity has been obtained, thanks to RossiXTE observations related to the physics of the matter in the innermost regions around compact objects (neutron stars and black holes) endowed with very strong gravitational fields [1]. In these objects the gravitational energy of accreting matter is converted very efficiently into radiation, mainly in the X-ray and $\gamma$-ray bands: the matter gradually spirals towards the compact object under the effect of viscous stress induced by layers of matter orbiting at different radii, downwards to the last stable orbit $\left(r_{\text {isco }}=6 G M / c^{2}\right.$ for a non-rotating black hole). Hence, by exploring these regions it is possible to investigate the properties of gravity in the strong-field regime and to measure parameters of collapsed objects such as the radius, mass and angular momentum. Different techniques, based on X-ray spectroscopy and X-ray timing, are very promising in this respect. For example, the detection of the relativistically broadened iron $K_{\alpha}$ fluorescence line in accreting black holes could lead to an estimation of the black hole spin if a model for the X-ray source and the accretion disk is assumed. A further in situ diagnostics of the strong gravity field is provided by fast X-ray timing measurements, in particular for what concerns the fast Quasi Periodic Oscillations (hereafter QPOs) that are present in the light curves of X-ray binary systems. The theoretical interpretation of these oscillations is still debated, however all models require at least one of the observed oscillation frequencies to be related to the azimuthal, orbital or Keplerian frequency of matter orbiting in the innermost disk regions [2]. Since the QPOs frequencies are likely related to the fundamental frequencies of motion, they allow for the estimation of the mass, spin and radius at which the signals are produced [3].

A significant progress in the understanding of the behavior of matter in the strongly curved spacetime near compact objects will therefore require long and deep X-ray timing studies, that could be guaranteed if future instrumentations with a collecting area larger than RXTE/PCA become operative in the next years. These experiments, with their higher counting statistics, would allow to study in greater detail variable phenomena on short time scales, as in the case of $\mathrm{kHz}$ QPOs. In this respect, it will be of crucial importance to reach the level of sensitivity needed to perform measurements of QPOs within a limited number of cycles, over which the individual oscillation trains remain coherent. Tracing the shape and recurrence times of the QPO trains is a critical step to discriminate among the different theoretical predictions of these phenomena $[4,5]$.

A higher sensitivity coupled with a good energy resolution may also lead to significant progress in the understanding of one of the most challenging problems in the physics of compact sources, i.e. the determination of the nuclear matter equation of state (EOS). This can be done by means of different observables that can provide independent measurements of the mass-radius relations: the $\mathrm{kHz}$ QPOs detections [3], the spectral fitting during thermonuclear explosions on the neutron star surface (type I X-ray bursts; [6]), the fitting of the pulse shape in millisecond X-ray pulsars [7]. Moreover, the study of seismic oscillation modes observed in the minutes-long decaying tail of magnetar giant flares (that reach a luminosity of $10^{42} \mathrm{erg} \mathrm{s}^{-1}$ ) seems to be very promising in the understanding of the neutron stars interior [8,9]. The lower frequencies of these oscillation modes likely arise from torsional shear oscillations of the crust [10]; by using them in combination with the magnetic field inferred for these magnetars it has been possible to rule out both very hard and very soft equations of state (as well as the EOS for strange stars). 
Several other scientific topics could be investigated with large area observatories: among them, the search for X-ray QPOs in magnetic white dwarf stars. These oscillations have been observed in the optical energy bands on a few seconds timescale [11], but no QPO has been observed so far in X-rays, perhaps due to the insufficient sensitivity of the present instruments.

In this paper we present the concept of a potential future mission devoted to X-ray timing studies, and whose principal aim is to investigate the physics underlying the behavior of compact objects. The paper is organized as follows: in Sect. 2 we present the Large Observatory For X-ray Timing (LOFT) mission concept, discussing its feasibility with modern technology; in Sect. 3 we present some simulations related to scientific topics adressed by LOFT, showing its performance; finally, in Sect. 4 we draw our conclusions.

\section{LOFT: mission concept}

With such scientific issues in mind, we aim at designing an X-ray timing observatory with a very large sensitive area (Large Area Observatory for X-ray Timing, LOFT). A natural question is how large this area has to be compared to the past missions. At this stage of the project we are mainly focused on the definition of a realistic mission with the largest possible area. The goal is to obtain a drastic improvement in the counting statistics with respect to previous missions. We are currently exploring the possibility to reach with reasonable costs an effective area which is about 20 times that of PCA $\left(0.6 \mathrm{~m}^{2}\right)$, that is in the range of a few tens of square meters.

However, the design of a mission devoted to deep timing studies of Galactic sources and transients is not only a matter of large area: several other factors have to be taken into account, such as the sensitivity, the spectral resolution, and the need of uninterrupted continuous pointings. These requirements give rise to many practical issues, mainly related to the volume, the power, the orbit attitude stability, the telemetry, the dead time, the construction time and — last but not leastthe costs. Up to now, the quest for timing experiments with tens of square meters sensitive area has been frustrated by the technical difficulties to solve most of these practical problems within the constraints of a space mission. A main issue has been the difficulty in manufacturing a very large number of detectors, while maintaining a high quality in all of them. Thanks to the significant improvement in the technology of Silicon detectors, we believe that most of these obstacles can be removed. In the following section, we discuss the feasibility of LOFT with the modern technology of Silicon Drift Detectors.

\subsection{The Silicon Drift Detector and the collimator}

In order to build an experiment with such a large area, it is crucial to employ a detector with high sensitivity but needing low resources in terms of absorbed electrical power, weight and cost. A very promising possibility is given by the use of the ALICE-D4 large-area Silicon Drift Detectors (SDDs) [14]. This kind of solid-state detectors decouple the collecting area to the sensitive area, allowing therefore to greatly reduce the electronic noise even at room temperature. Their working principle is the following: a photon absorbed in the Silicon bulk creates an electron cloud, which is then drifted by an applied electric field towards an array of collecting anodes; the signal recorded at the anodes allows to measure both the photon energy and its absorption location. The X-ray spectroscopic performance of these detectors, developed by INFN-Trieste in collaboration with, 
and manufactured by, Canberra Inc., as particle trackers for the ALICE experiment at the Large Hadron Collider (CERN, Geneva), have been investigated at the INAF/IASF-Roma X-ray facility $[15,16,17]$. The basic detector (Figure 1) is $7.56 \mathrm{~cm} \times 8.26 \mathrm{~cm}$, for an active area of $53 \mathrm{~cm}^{2}$ and a thickness of $300 \mu \mathrm{m}$; each half of the detector is read-out by 256 anodes (pitch distance $294 \mu \mathrm{m}$ ).

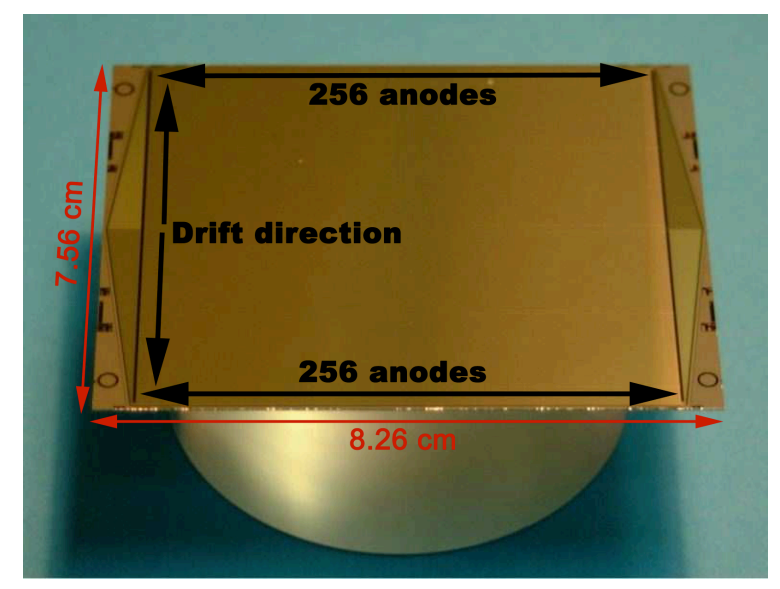

Figure 1: The ALICE-D4 Silicon Drift Detector.

Despite a detector design not optimized for the X-ray applications, very promising results have been obtained: an energy resolution of 300-500 eV FWHM in the 2-10 keV band has been reached at room temperature, and a lower energy threshold below $2 \mathrm{keV}$ has been demonstrated feasible. The high-energy sensitivity threshold depends mainly on the thickness of the Silicon bulk, which can be increased up to $1 \mathrm{~mm}$ in future productions. Moreover, the mass production of these detectors has been already demonstrated (about 280 SDDs are currently successfully operating at LHC, for a total active area of $\sim 1.5 \mathrm{~m}^{2}$ ) with an high quality yield. The timing resolution is limited by the drift time, and is of a few $\mu$ s. A revised detector design (optimized for the X-ray spectroscopy) and a custom-made front-end ASIC are currently in production and testing in our laboratories. The overall power consumption will be lowered to the level of about $1 \mathrm{~mW} /$ channel.

In order to collimate the incoming X-rays and to restrict the field of view to around 1 degree, an interesting possibility, that limits both the weight and the dimensions of the collimator, is to use lead-glass microcapillary plates (MCPs). The collimating performance of a MCP with a thickness of about $1 \mathrm{~mm}$, an hole size of $25 \mu \mathrm{m}$ and an open-area ratio of $\sim 70 \%$ (corresponding to a hole-tohole distance of $28 \mu \mathrm{m}$, and to an aperture angle of $1.4^{\circ} \mathrm{FWHM}$ ) has been investigated by means of GEANT-4 MonteCarlo simulations. The results (Figure 2) demonstrate that the collimation is very good up to about $30 \mathrm{keV}$, beyond which the Silicon efficiency however decreases significantly. Experimental tests to validate the simulations are envisaged for the next months.

\subsection{Experiment design}

A sketch of our mission design is shown in Figure 3. Using a standard PRIMA-Science satellite platform (Thales Alenia Space, Italy) and a Vega launcher, the arrays of SDDs and collimators can be deployed exploiting the solar panels technology. A total geometrical area of the detectors up to $32 \mathrm{~m}^{2}$ could be allocated, but power constraints will likely limit the simultaneous operations to an area of $\sim 23 \mathrm{~m}^{2}$. Upon considering the open area ratio of the collimators and the "dead" zones 


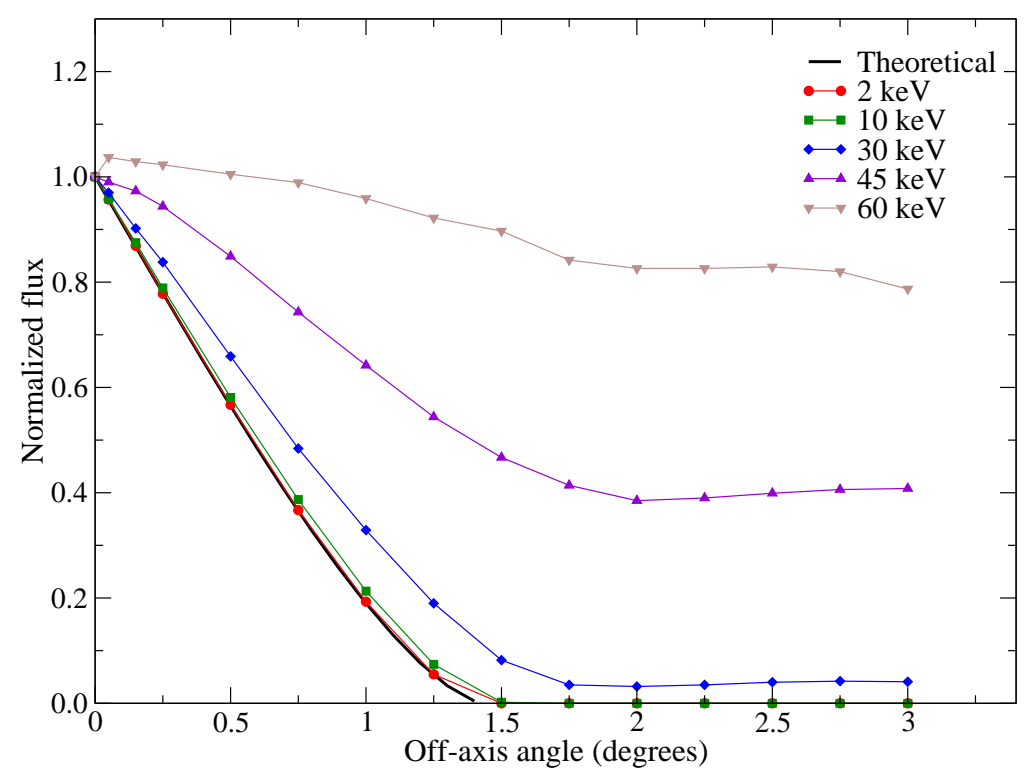

Figure 2: Results of the MonteCarlo simulations of the lead-glass microcapillary plate collimation. In the plot is shown the normalized response as a function of the off-axis angle, for various energies. The thick black line represents the response of an ideal collimator. It is evident how the collimation is very good up to about $30 \mathrm{keV}$.

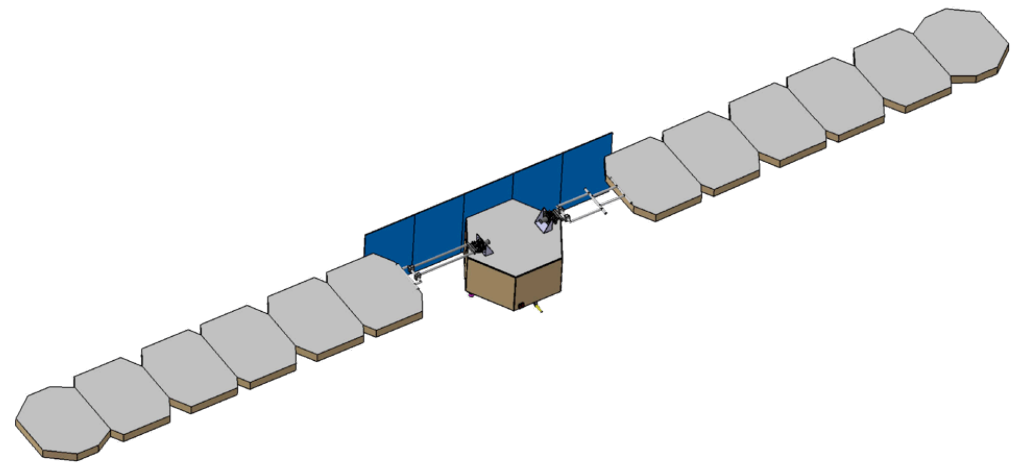

Figure 3: The LOFT experiment concept: the spacecraft and the panels of SDD arrays with their collimators are shown. The ASM is not included in this picture.

of the detectors, an active area of $13 \mathrm{~m}^{2}$ can be reached. The total mass of the satellite is about $1300 \mathrm{~kg}$. An estimate of the weight of the detectors, the interface electronics, the collimators and the array frame is around $6 \mathrm{~kg} \mathrm{~m}^{-2}$. We envisage also the addition to the payload of a SDD-based wide-field coded mask X-ray All Sky Monitor (ASM), with asymmetric 2D position reconstruction capabilites, a total fully coded field of view of the order of 2 steradians and an angular resolution of a few arcmin. 


\section{Expected performance}

The $13 \mathrm{~m}^{2}$ LOFT effective area results in a 2-30 keV sensitivity of about $10 \mathrm{mCrab}$ (at $5 \sigma$ in $1 \mathrm{~s}$ ), i.e. $0.05 \mathrm{mCrab}$ in 1 day. In what follows, we are going to quantify this sensitivity in terms of the scientific performance of the experiment, showing the performance of LOFT with respect to the previous instruments, in particular the RXTE/PCA.

For what concerns coherent signals, it is interesting to simulate what is the limit pulsed fraction detectable by LOFT over different time scales. This is a crucial issue in the interpretation of the recent discovery of transient pulsations in Low Mass X-ray Binary systems, as in the case of Aql $\mathrm{X}-1$, where a coherent millisecond X-ray pulsation $(\sim 550 \mathrm{~Hz})$ was found by Casella et al. [12] in a data segment of a few hundred seconds, at constant frequency, over the whole archival RXTE/PCA dataset. The interpretation of similar phenomena as well as the lack of pulsations in other LMXBs is still debated, and different possibilities have been explored so far: the transient pulsation may be due to some scattering or screening medium that occasionally disappears or strongly reduces its optical thickness, or it may be caused by a rare anisotropy on the neutron star surface. The significant improvement provided by LOFT's counting statistics with respect to RXTE/PCA could provide the possibility to explore the behavior of this source and other LMXBs on shorter time scales, with the capability to detect low pulsed fractions, offering a large dataset to test the different theoretical interpretations.

Aql X-1 $550 \mathrm{~Hz}$ pulsations with p.f. $0.5 \%$

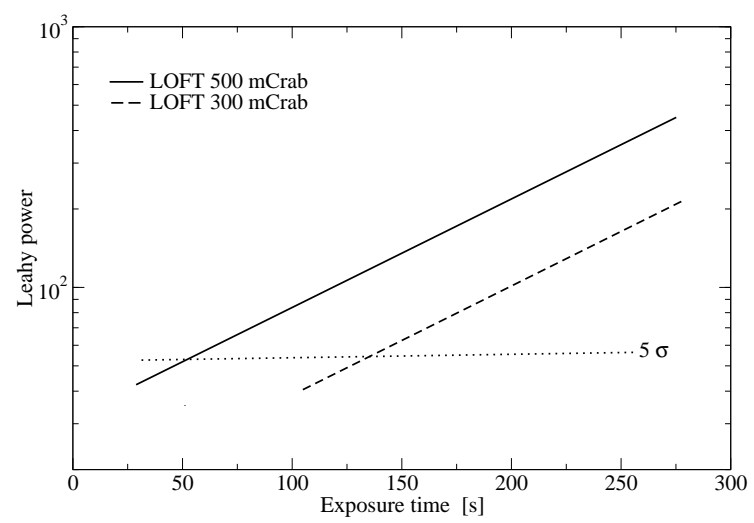

Aql X-1 $550 \mathrm{~Hz}$ pulsation, $128 \mathrm{~s}$ observation

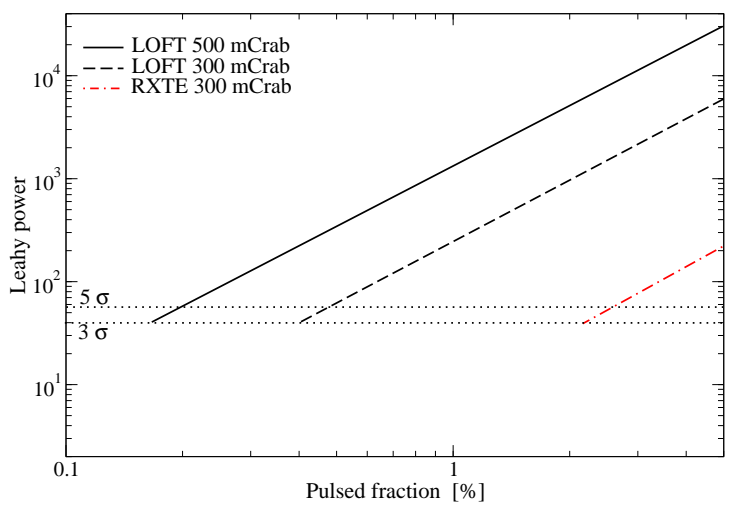

Figure 4: Simulations of the LOFT performance for observations of Aquila X-1. Left panel: the limit pulsed fraction detectable in a 128 s observation. RXTE (red line) and LOFT (black line) simulations rates are shown, the latter for a source rate of 300 and $500 \mathrm{mCrab}$. Right panel: Minimum exposure time needed to detect coherent pulsation with a pulsed fraction of $0.5 \%$ with LOFT.

In Figure 4 (left panel), we show a comparison between the limit pulsed fraction detectable by LOFT (black line) and RXTE/PCA (red line) in a time scale of $128 \mathrm{~s}$. In particular, by assuming a pulsation frequency of $550 \mathrm{~Hz}$ and a rate of $63000 \mathrm{cts} / \mathrm{s}$ for LOFT $(\sim 300 \mathrm{mCrab})$, we show the pulsed fraction detectable at a significance level above 3 and $5 \sigma$ (taking into account the total number of trials). It is worth noticing that we will be able to explore pulsed fractions which are about one order of magnitude lower that the ones detectable or detected so far by past experiments. We now estimate what is the lowest time scale on which we may detect a fixed pulsed fraction. 
Assuming a pulsed fraction of $0.5 \%$ for a source of $\sim 300 \mathrm{mCrab}$, we find that this pulsation is detectable on a time scale of $\sim 120 \mathrm{~s}$ at a significance level of $5 \sigma$ (Figure 4, right panel), decreasing to $\sim 50 \mathrm{~s}$ if the flux increases to $500 \mathrm{mCrab}$. The capability to reach a significant detection on these short time scales will be very useful e.g. for the study of close binary systems: high counting statistics on short time scale will avoid the Doppler smearing (orbital time scale) and will allow to investigate fast dynamics.

The high sensitivity of LOFT would also allow to follow the time evolution of individual oscillation modes observed during the decaying tails of a magnetar flare (intermediate or giant flare). LOFT is planned as a pointed observatory, thus the chances to detect on-axis these events are low. On the other hand, these extremely high-flux and hard events can be seen off-axis through the collimator, still getting a high count rate, as happened to the RXTE/PCA. Hence, we have simulated the oscillation modes observed in the light curve of the decaying tail of the SGR 180620 giant flare [8, 9] for both LOFT and RXTE.
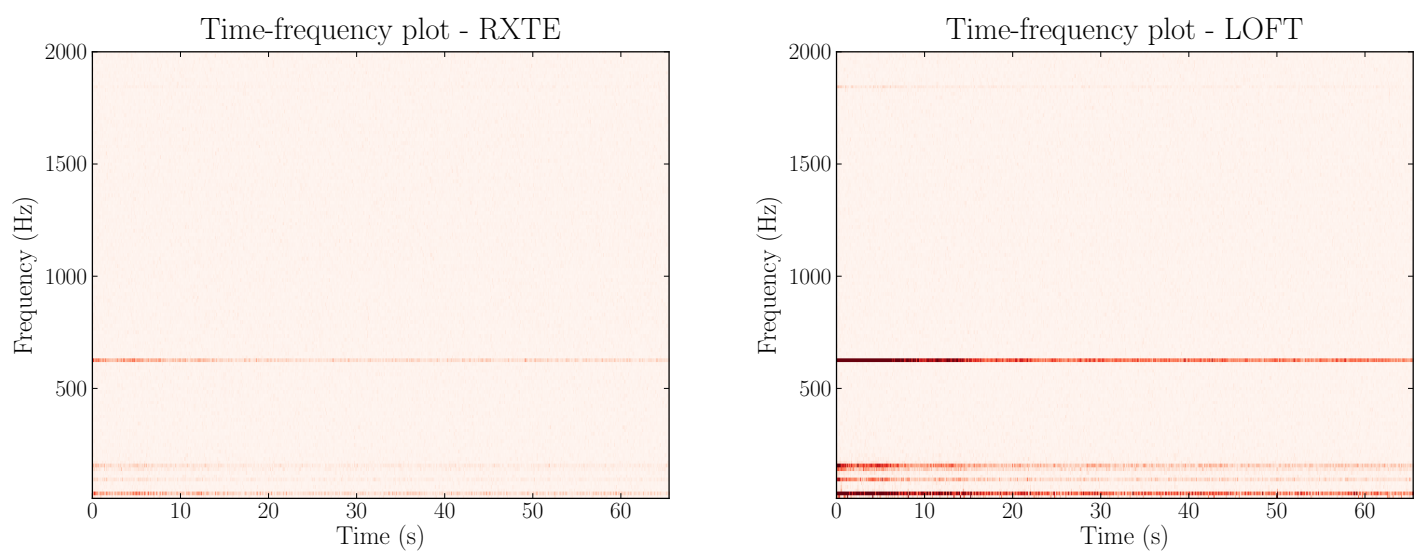

Figure 5: Simulation of the oscillation modes in the decaying tail of the SGR 1806-20 giant flare, as seen by RXTE (left panel) and LOFT (right panel), in a 64 s-long observation. Time-frequency plot, with power spectra calculated on a $64 \mathrm{~ms}$ timescale. The color scale is the same for the two plots.

We simulated a $64 \mathrm{~s}$ observation with $250 \mu$ s time resolution, assuming an intensity of 1700 Crab, as estimated for the hyper-flare in 2004 , seen at $30^{\circ}$ off-axis for both instruments (for RXTE we adopted the count rate reported in [8]). We reproduced the following oscillation mode frequencies: $0.132,18,26,29,92,150,625,1840 \mathrm{~Hz}$, which correspond to pulsed fractions of $0.5,0.04$, $0.05,0.2,0.1,0.17,0.2,0.05$, as reported in [9]. In Figure 5, we show the comparison between the time-frequency behavior of the decaying tails as followed by LOFT (right panel) and RXTE (left panel). In both plots, a power-spectrum density has been extracted every $64 \mathrm{~ms}$. It is evident that the high sensitivity of LOFT will allow to follow the dynamics of such an event, with the onset of various oscillation modes with different frequencies and pulsed fractions, in time scales of some tens of milliseconds.

As an example of LOFT performance with respect to non-coherent signals, in Figure 6 is shown the power spectral density obtained by simulating a 1024 s observation of the Galactic blackhole binary GRS 1915+105, in a state when this source showed two main QPOs at 40 and $67 \mathrm{~Hz}$ [13], with a hint of a further QPO at $56 \mathrm{~Hz}$. Besides these three QPOs, we added in the simulation 


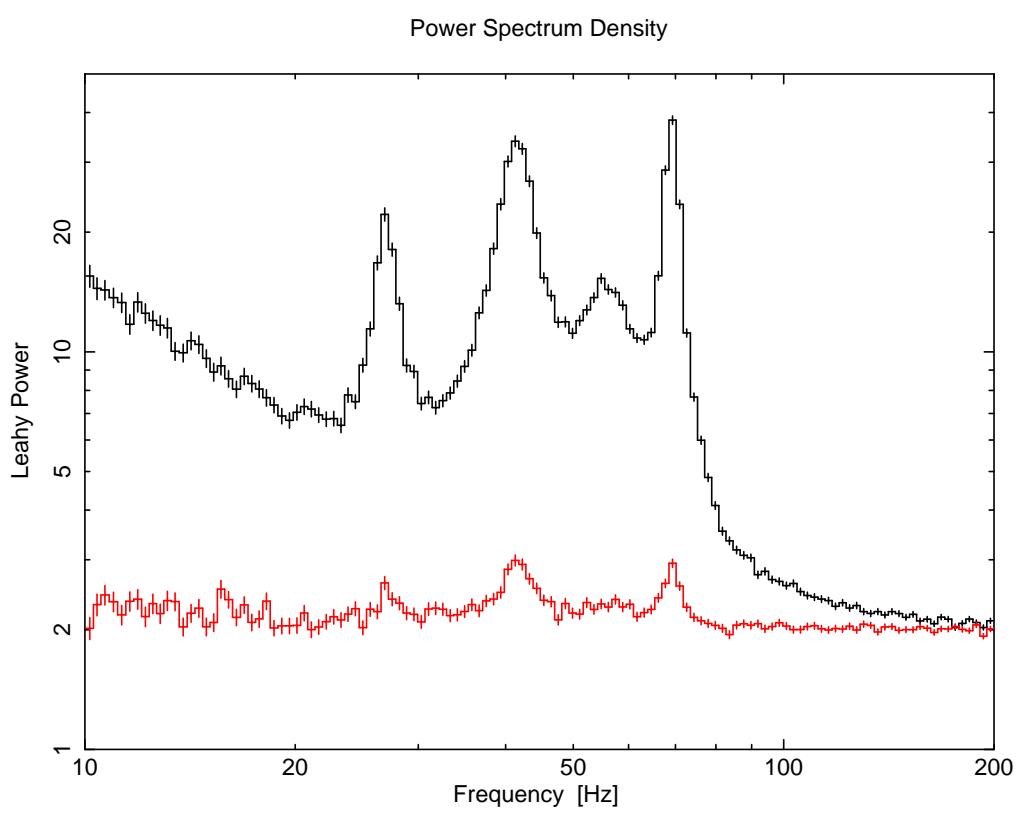

Figure 6: Power spectrum of a LOFT (black line) and RXTE (red line) simulated 1024 s observation of the microquasar GRS 1915+105.

a further, fainter oscillation at $27 \mathrm{~Hz}$, to show the dramatic increase in the QPO detectability given by the much higher counting statistics of LOFT with respect to the RXTE/PCA. The detection and the precise determination of the QPO parameters will allow to investigate in detail their relation to the various source states, linked to the physics of the accretion around the compact object, and to discriminate between the various models.

\section{Conclusions}

Follow-up instruments to the RXTE/PCA are needed to further improve the understanding of the physics in the strong gravitational fields present in the close proximity of black holes and neutron stars. For this reason, in the recent years several experiments and missions devoted to X-ray timing have been proposed. Among them, the Indian mission ASTROSAT [18], the HTRS instrument onboard IXO (W. Becker, these proceedings), and the AXTAR mission (R. Remillard, these proceedings). In this paper we have presented the mission concept for a large area observatory for X-ray timing studies, LOFT. The main difference between LOFT and the other proposed experiments lies in its huge effective area of $13 \mathrm{~m}^{2}$ (versus approx. $0.6,3$, and $4 \mathrm{~m}^{2}$ for ASTROSAT/LAXPC, IXO/HTRS and AXTAR, respectively, however covering very different sensitivity bands), now achievable thanks to the considerable progresses done in the technology of Silicon Drift Detectors, which allow also to reach a good energy resolution (300-500 eV FWHM) even at room temperature. 
LOFT, being a mission dedicated to timing studies, will provide a crucial amount of observational time for several interesting targets. This fact marks a significant difference between LOFT and a multi-purpose observatory like IXO. In fact, the latter will have only a small fraction of time allocated for timing studies with the HTRS instrument. However, the two experiments may be considered complementary: LOFT is best suited to the study of bright sources, for which the relatively high background contamination is not a problem, while IXO/HTRS, on the other hand, will optimize the study of weak sources for which a low background is required.

\section{References}

[1] D. Psaltis, Living Reviews in Relativity, 11, 9, 2008.

[2] L. Stella, in Physics of Relativistic Objects in Compact Binaries, Springer Netherlands, 2009.

[3] M. Van Der Klis, in Compact Stellar X-ray Sources, Cambridge University Press, 2006.

[4] L. Stella, M. Vietri \& S. Morsink, ApJL, 524, 63, 1999.

[5] M. A. Abramowicz \& W. Kluźniak, A\&A, 374, L19, 2001.

[6] J. Cottam et al., Nature, 420, 51, 2002.

[7] D. A. Leahy et al., ApJ, 691, 1235, 2009.

[8] G. L. Israel et al., ApJ, 628, L53, 2005.

[9] T. E. Strohmayer \& A. L. Watts, ApJ, 653, 593, 2006.

[10] R. C. Duncan, ApJ, 498, L45, 2008.

[11] B. Warner, PASP, 116, 115, 2004.

[12] P. G. Casella et al., ApJ, 674, L41, 2008.

[13] T. E. Strohmayer, ApJ, 554, L169, 2001.

[14] A. Vacchi, et al., Nucl. Meth. Instr. A, 306, 187, 1991.

[15] G. Zampa et al., IEEE Trans. Nucl. Science, 56, 832, 2009.

[16] G. Zampa et al., Nucl. Meth. Instr. A, in preparation, 2010.

[17] R. Campana et al., Nucl. Meth. Instr. A, in preparation, 2010.

[18] P. C. Agrawal, Advances in Space Research, 38, 2989, 2006. 